\title{
iSim: Uma Estratégia de Agrupamento de Usuários para Descarregamento de Dados em Redes Móveis Centradas na Informacão
}

\author{
Adriana Viriato Ribeiro ${ }^{1}$, Leobino N. Sampaio ${ }^{1}$ (Orientador) \\ ${ }^{1}$ Programa de Pós Graduação em Ciência da Computação (PGCOMP) \\ Universidade Federal da Bahia (UFBA) \\ Salvador - BA - Brasil \\ \{adrianavr, leobino\}@ufba.br
}

\begin{abstract}
This paper presents iSim, a high-affinity similarity measure to group users based on content diversity and frequency requests. We designed the proposed strategy in an Information-Centric Cellular Network to provide data offloading. Experimental results show the iSim's adaptability to different scenarios and present best results in hit ratio and data offloading when the strategy is used. Futhermore, the results also show that hit ratio and data offloading is better when similarity is based in both content diversity and frequency of common requests than when just one factor is used.
\end{abstract}

Resumo. Este artigo apresenta a iSim, uma estratégia de cálculo de similaridade e agrupamento entre usuários baseada na diversidade e na frequência dos conteúdos requisitados. A estratégia foi aplicada a um cenário de Redes Móveis Centradas na Informação, com o objetivo de prover descarregamento de dados. Resultados experimentais demonstram a capacidade de adaptação da estratégia proposta a diferentes cenários e evidenciam melhoras na taxa de acerto e no descarregamento de dados quando a estratégia é utilizada. Além disso, foi possível observar que quando a similaridade é baseada tanto na quantidade de conteúdos em comum quanto na frequência, as taxas de acerto e de descarregamento de dados são superiores a quando apenas um fator é utilizado.

\section{Introdução}

Em decorrência da inadequação na arquitetura da Internet atual em lidar com desafios advindos da popularização de redes móveis e de aplicações famintas por banda, além da mudança no perfil dos usuários, algumas propostas sugerem o uso do paradigma de Redes Centradas na Informação (do inglês, Information-Centric Networks - ICN) em cenários de densificação de redes. Assim, trabalhos tem sido desenvolvidos considerando o uso de cache em pequenas estações bases (do inglês, Small-Cell Base Stations - SCBS) para armazenar conteúdos e deixá-los mais próximos dos consumidores finais, reduzindo a quantidade de requisições na estação principal (do inglês, Macro Base Station - MBS) e fornecendo descarregamento de dados [ElBamby et al. 2014, Pantisano et al. 2015, Chen and Kountouris 2015, Hajri and Assaad 2016].

Embora a inclusão de cache nos equipamentos de rede seja uma alternativa para deixar o conteúdo mais próximo do usuário, é preciso definir estratégias que potencializem seu uso. Desta forma, pesquisas mais recentes têm sugerido o uso de técnicas 
cientes de contexto [Chang et al. 2016] e técnicas de agrupamento com o objetivo de reunir usuários com base em características similares e associá-los às SCBS mais apropriadas [Ioannou and Weber 2016].

Apesar do uso de agrupamento apresentar ganhos nas taxas de acerto, os trabalhos que recorrem a esta estratégia [ElBamby et al. 2014, Pantisano et al. 2015] observam apenas a frequência dos conteúdos requisitados na definição dos grupos. O problema, contudo, é que a frequência dos conteúdos não relata de forma precisa o nível de afinidade entre os usuários. A afinidade deve corresponder à aproximação entre os usuários do ponto de vista das suas preferências em relação aos conteúdos disponíveis na rede. Como a avaliação da frequência considera apenas os conteúdos solicitados em comum, muitas vezes ela pode se tornar ineficiente ao determinar usuários afins. Assim, dois consumidores podem ter interesse por um único conteúdo em comum e, ainda assim, serem considerados similares em decorrência da frequência de solicitações e da função de similaridade utilizada. Por exemplo, tendo dois consumidores A e B cujos conteúdos solicitados são $C_{a}=\{t, s, z\}$ e $C_{b}=\{l, w, z\}$ e as frequências de solicitação são $F_{a}=\{1,4,1\}$ e $F_{b}=\{2,5,1\}$, considerando apenas a frequência dos conteúdos solicitados em comum (conteúdo $z$ ) e calculando a afinidade com o cosseno, eles teriam similaridade máxima. Notoriamente, tal similaridade pouco reflete a afinidade entre os consumidores.

Assim, esse trabalho visa resolver o seguinte problema: como melhorar o descarregamento de dados em redes de cache considerando a afinidade entre os usuários para formação dos agrupamentos. Para isso, foi proposta a iSim (interests' Similarity), uma estratégia ciente de contexto que explora a formação de grupos de usuários similares em uma rede celular de pequeno porte (do inglês, Small-Cell Networks - SCN) centrada na informação com o intuito de melhorar o descarregamento de dados na MBS, levando em consideração a diversidade dos conteúdos requisitados pelos usuários e a frequência de solicitações. O desenvolvimento da estratégia proposta envolveu a formulação de uma equação de similaridade para um cenário de Redes Centradas na Informação aplicado às Redes Móveis com o intuito de fazer descarregamento de dados. Portanto, as principais contribuições deste trabalho são:

- Avaliação do impacto da utilização de Redes Centradas na Informação para auxiliar no problema de descarregamento de dados em Redes Móveis.

- Desenvolvimento de ambiente de simulação composto por uma Small Cell Network com uma arquitetura baseada em Redes Centradas na Informação.

- Desenvolvimento de uma função de similaridade baseada no Índice de Jaccard e na Similaridade do Cosseno e de um algoritmo de agrupamento baseado no kNN (k-Nearest Neighbor).

- Avaliação experimental da iSim considerando diferentes fatores e parâmetros.

As demais seções desse artigo estão organizadas da seguinte forma: na Seção 2 são discutidos os principais trabalhos relacionados, na Seção 3 é definida a estratégia proposta, na Seção 4 são discutidos os principais resultados obtidos e na Seção 6 são descritas as publicações relacionadas ao trabalho.

\section{Trabalhos Relacionados}

Os trabalhos de [ElBamby et al. 2014, Pantisano et al. 2015] atuam em cenários de SCN com o intuito de prover descarregamento de dados e melhorias em relação à utilização da 
rede através de abordagens ciente de contexto. Ao comparar o cenário, objetivo, contexto utilizado, função de similaride, entre outras características, é possível observar que o trabalho de [Pantisano et al. 2015] diferencia-se da iSim em relação ao objetivo principal, que envolve melhoria na alocação de banda, no QoS e na redução de retransmissões. No entanto, o descarregamento de dados também pode ter influência em alguns desses aspectos, principalmente na melhoria do QoS, visto que diminui a sobrecarga das estações principais. Uma outra característica observada é que o trabalho de [Pantisano et al. 2015] é ciente de cache e determina uma função própria que se baseia nas frequências de requisições dos vizinhos em relação ao conteúdo para calcular a similaridade entre os usuários.

O trabalho de [ElBamby et al. 2014], embora assemelhe-se mais à iSim, possui como principal diferença a função de similaridade utilizada, visto que a iSim propõe uma soma ponderada entre dois aspectos - diversidade e frequência - ao passo que [ElBamby et al. 2014] considera apenas a frequência das requisições. Além disso, os algoritmos de agrupamento utilizados são distintos e em [ElBamby et al. 2014] é criada uma estratégia própria para realizar o gerenciamento de cache.

\section{3. iSim: interests' Similarity}

A iSim é uma estratégia ciente de contexto que visa utilizar a afinidade dos usuários em relação aos interesses por conteúdo para agrupá-los e associá-los, de forma lógica, à diferentes caches com a intenção de aumentar a taxa de acerto nos caches locais. $\mathrm{O}$ aumento da taxa de acerto é decorrente do aumento da probabilidade de usuários agrupados requisitarem os mesmos conteúdos, devido a afinidade entre eles. Com o aumento da taxa de acerto nos caches locais, é possível diminuir a quantidade de requisições à estação principal, culminando no descarregamento de dados.

Essa estratégia pode ser aplicada e adaptada a diferentes cenários. Neste trabalho, ela foi associada a um cenário de Redes Móveis Centradas na Informação, que pode ser observado na Figura 1. O cenário é composto por três entidades principais: os dispositivos móveis (DMs), que representam os equipamentos dos usuários, as estações adicionais, representadas pelas SCBS com cache, e a estação principal ou MBS. Cada uma dessas estruturas executa diferentes papéis na rede.

Os DMs representam os usuários e são responsáveis por fazer as requisições para os conteúdos. A SCBS trata-se de uma estação secundária composta por um cache de capacidade de armazenamento inferior à MBS, capaz de manter uma cópia local de uma porcentagem do total de conteúdos. Já a MBS é a estação principal composta por um cache capaz de armazenar todos os conteúdos e é responsável pela execução da iSim.

Quando um DM faz uma requisição por um conteúdo, a SCBS e a MBS podem usar dois tipos de comunicação, a comunicação direta ou a comunicação indireta. Conforme Figura 1, na comunicação direta, quando o conteúdo não é encontrado no cache local, a SCBS requisita diretamente à MBS. Enquanto na comunicação indireta, todas as SCBS são percorridas antes de alcançar a MBS.

Além dos equipamentos que compõem a rede móvel e dos tipos de comunicação entre eles, algumas estruturas adicionais são criadas para suportar uma arquitetura centrada na informação e para dar suporte à estratégia. As estruturas utilizadas foram: Tabela de Interesses Pendentes (do inglês, Pending Interest Table - PIT), que mantém os interesses que ainda não foram resolvidos; a cache ou Content Store, que contém cópia de 


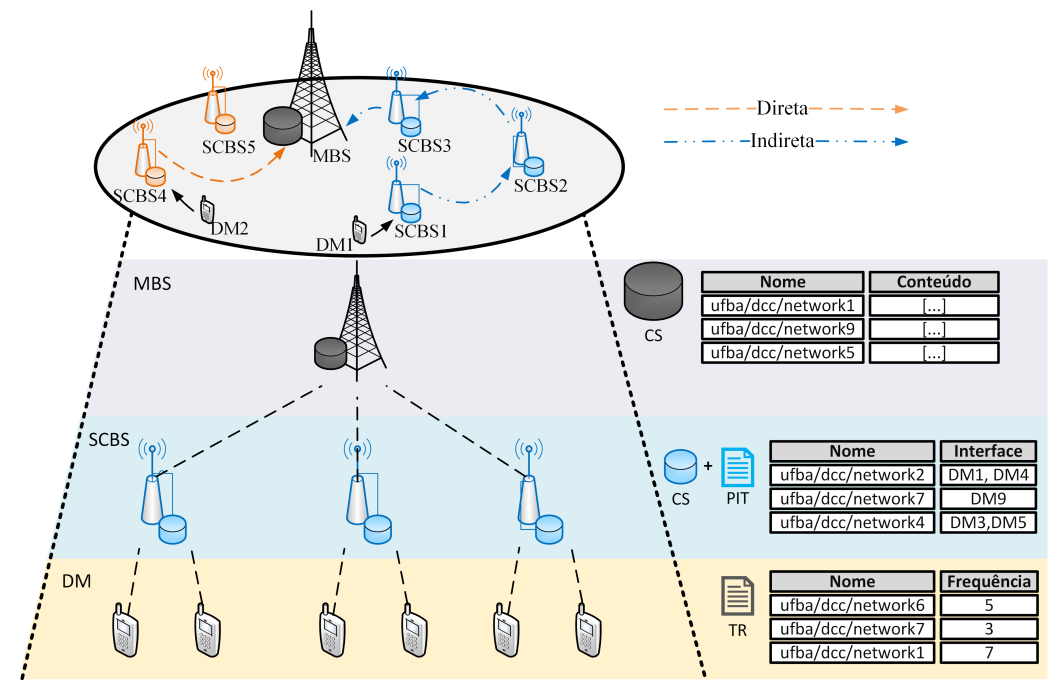

Figura 1. Exemplo de Rede Móvel Centrada na Informação na qual a iSim pode ser utilizada.

conteúdos; e a Tabela de Requisições (TR), que armazena os conteúdos solicitados e a frequência de solicitação para cada usuário.

Uma vez descrito o cenário utilizado, é possível observar o funcionamento da iSim. Para calcular a afinidade entre os usuários são utilizadas duas medidas de similaridade: o Índice de Jaccard e a similaridade do cosseno. Dessa forma, a similaridade entre os usuários é medida através da Equação 1. A primeira parte representa o Índice de Jaccard e foi utilizada para medir a similaridade de acordo com os conteúdos que dois nós $(i, j)$ tem interesse. Já na segunda parte tem-se o cálculo da lei de similaridade do cosseno em relação à frequência de requisições de conteúdos em comum.

$$
S_{i, j}=\beta\left(\frac{\left|C_{i} \cap C_{j}\right|}{\left|C_{i} \cup C_{j}\right|}\right)+(1-\beta)\left(\frac{F_{i} \cdot F_{j}}{\left\|F_{i}\right\|\left\|F_{j}\right\|}\right)
$$

Desta forma, $C_{i}$ e $C_{j}$ representam os conjuntos de conteúdos que foram solicitados por $i$ e por $j$, respectivamente. Sendo que, durante o cálculo da similaridade é utilizada a quantidade de elementos nos conjuntos interseção e união, não os conjuntos propriamente ditos; $F_{i}$ e $F_{j}$ representam a frequência, em $i$ e em $j$, em que os conteúdos comuns foram requisitados; e $\beta$ define um peso entre as partes da equação e permite que seja avaliado o impacto delas no cálculo de similaridade. A utilização do parâmetro $\beta$ permite flexibilidade e adaptabilidade no cálculo da similaridade, visto que possibilita o aumento ou diminuição do impacto das partes da equação de acordo com as características do cenário.

A iSim calcula a similaridade de forma centralizada na MBS para formar $G$ clusters, onde $G$ é a quantidade de SCBS. Com esse propósito, o processo de agrupamento de usuários é iniciado quando a MBS recebe a Tabela de Requisições de todos os usuários para construir uma matriz $M=\left[m_{u, c}\right]$ de requisições de conteúdo, para os conjuntos $u=\{1,2, \ldots, n U\}$ e $c=\{1,2, \ldots, n C\}$, assumindo que $n U$ e $n C$ representam o número de usuários e de conteúdos na rede, respectivamente. Assim, cada elemento $m_{u, c}$ representa o número de requisições por conteúdo para cada usuário. A MBS utiliza a matriz $M$ como 
entrada para calcular a similaridade entre os usuários para cada elemento $m_{u, c}$. Os resultados obtidos desse cálculo são utilizados para construir uma matriz simétrica $U=\left[u_{i, j}\right]$, para $i=\{1,2, \ldots, n U\}$ e $j=\{1,2, \ldots, n U\}$, na qual, $u_{i, j}=u_{j, i}$, na qual cada elemento $u_{i, j}$ contém a similaridade entre os usuários $i$ e $j$. Em seguida, é iniciado o processo de agrupamento que classifica os usuários em grupos de acordo com suas similaridades.

Para realizar o agrupamento foi utilizado o algoritmo kNN. Para aplicação do kNN, a iSim calcula a média de similaridade entre um nó e seus vizinhos. O nó com maior média é definido como líder do agrupamento. Assim, a formação do agrupamento é realizada com o nó líder e seus k vizinhos mais próximos. Quando um grupo é estabelecido, os nós que pertencem a ele são removidos de $U$ e o processo é repetido até que todos os agrupamentos sejam formados. Depois dessa etapa, a MBS envia uma mensagem aos nós indicando a qual cache eles devem se associar.

\section{Experimentos e Resultados}

Foram realizados experimentos para avaliar o desempenho da iSim de acordo com diferentes fatores, como variação nos pesos da equação, tamanho do cache, distribuição de Zipf, quantidade de dispositivos e de conteúdos, entre outros. Esta seção apresenta uma análise dos principais resultados obtidos.

A Figura 2 expõe o comportamento da taxa de acerto em relação ao uso da função de similaridade e da variação do tamanho do cache. Nessa figura é possível observar a influência das variações da equação de similaridade, bem como os efeitos do cenário de agrupamento randômico, também chamado de sem similaridade. Em relação ao uso da equação de similaridade, para todos os tamanhos de cache $(10,20,30)$, é possível observar que quando apenas a quantidade de conteúdos em comum é levada em consideração $(\beta=1)$, a taxa de acerto é superior a quando apenas a frequência é utilizada $(\beta=0)$. Isso pode ser justificado devido ao fato de que ao contabilizar apenas a frequência, é possível que dois usuários sejam considerados similares mesmo tendo um único conteúdo em comum. Dessa forma, como o Índice de Jaccard considera a relação entre os conteúdos requisitados pelos dois nós, os agrupamentos baseados nessa relação de similaridade conseguem alcançar resultados superiores. Apesar disso, os melhores resultados foram obtidos quando ambos foram utilizados $(\beta=0.5)$, evidenciando a importância de uma equação de similaridade que considere esses dois fatores.

A distinção entre as taxas de acerto dos gráficos das Figuras 2(a) 2(b) 2(c) se dá devido ao aumento do tamanho do cache. Desta forma, é possível observar que há um crescimento constante proporcional à capacidade de armazenamento. Sendo que, quando utilizada a equação de similaridade, as taxas de acerto conseguem atingir, aproximadamente, o dobro da capacidade de armazenamento. Em decorrência dessa melhora, ao relacionar os tamanhos do cache 10 e 20, conforme a Figura 2(d), observa-se que a taxa de acerto obtida sem similaridade com um cache $=20$ alcança valores próximos ao melhor valor obtido com uso de similaridade em um cache $=10$. Ao comparar os caches de tamanho 20 e 30, percebe-se que com o uso da similaridade em um cache $=20$ obtém-se valores superiores ao cenário sem similaridade em um cache $=30$. Isso evidencia que a utilização de agrupamentos que consideram a similaridade dos usuários torna, de fato, o gerenciamento de cache mais eficiente e possibilita economia de recursos, visto que os mesmos valores de taxa de acerto podem ser obtidos com caches de tamanho inferior. 


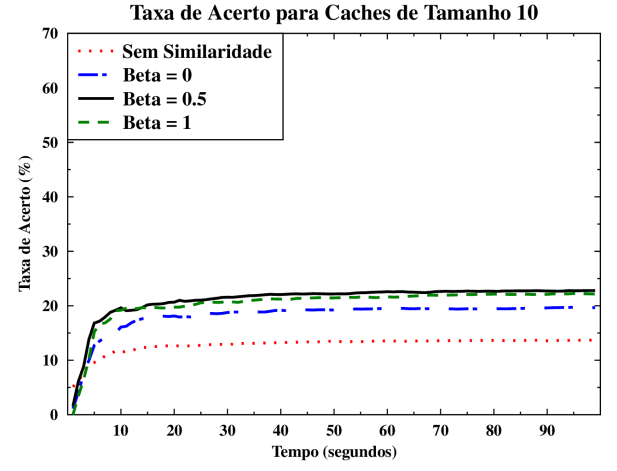

(a)

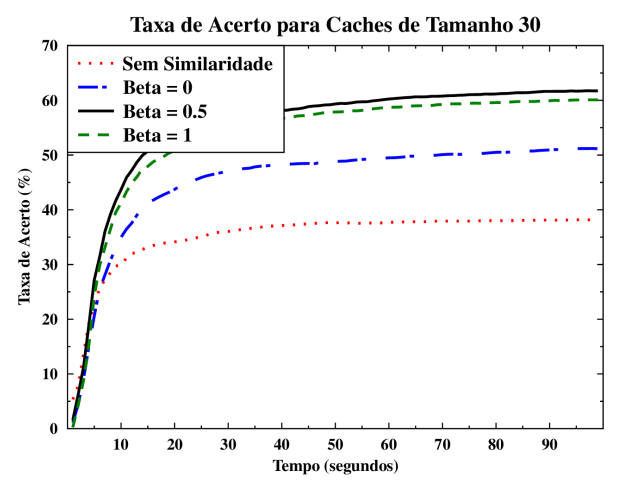

(c)

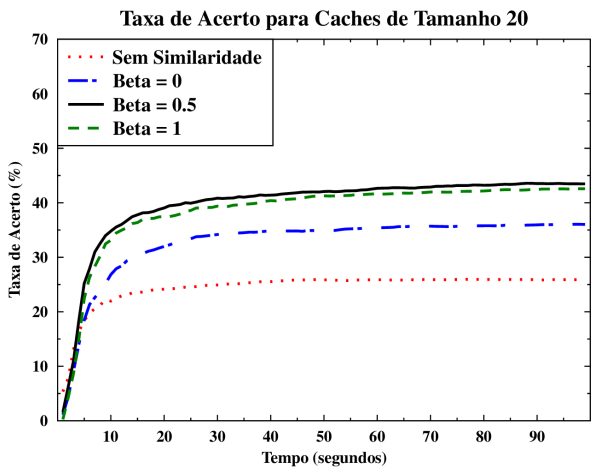

(b)

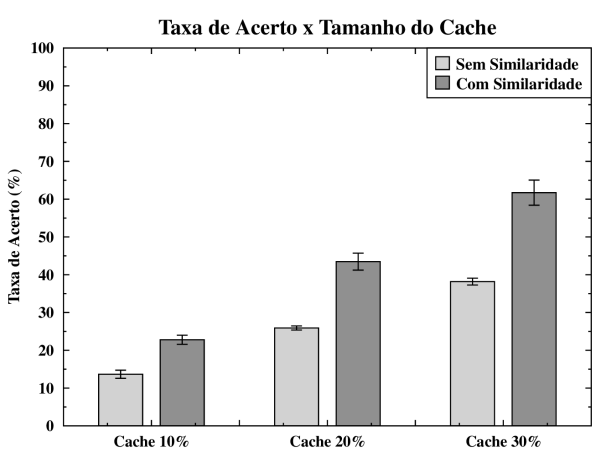

(d)

Figura 2. Relação entre o Tamanho do Cache e a Taxa de Acerto.

Ao avaliar o efeito da variação do alpha na taxa de acerto, nota-se que mesmo com diferentes valores para o parâmetro Zipf, o comportamento da função de similaridade permanece o mesmo: os resultados com valor de $\beta=0.5 \mathrm{ou} \beta=1$ são superiores a quando considera-se apenas a frequência. No entanto, os efeitos do cálculo de similaridade são baixos. Isso acontece porque quando o parâmetro Zipf é menor, há um pequeno conjunto de conteúdos populares que, consequentemente, são requisitados mais vezes. Como a política de descarte utilizada é a LFU, os conteúdos mais populares ficam armazenados por mais tempo, satisfazendo as requisições e culminando no aumento da taxa de acerto, independente da estratégia de agrupamento utilizada.

Na Figura 3(a) é possível observar o comportamento entre a similaridade e os tipos de comunicação em relação à taxa de descarregamento. Apesar de a taxa de acerto e de descarregamento de dados serem, comumente, proporcionais uma a outra, em cenários com comunicação indireta é possível observar comportamento oposto. De acordo com a Figura 3(a), a taxa de descarregamento em um cenário sem similaridade e com comunicação direta é de $22 \%$. Esta melhora é decorrente da adição das três SCBS, cada uma com cache de tamanho 20. Ainda na Figura 3(a), o cenário com comunicação direta e com similaridade consegue obter resultados superiores ao cenário com comunicação indireta e sem similaridade, evidenciando que a utilização de agrupamentos influencia mais no descarregamento do que a forma de comunicação.

Os melhores resultados para descarregamento de dados são aqueles obtidos quando os grupos são formados de acordo com a similaridade dos nós e quando todas 
as SCBS são percorridas antes de buscar na estação central (comunicação indireta).

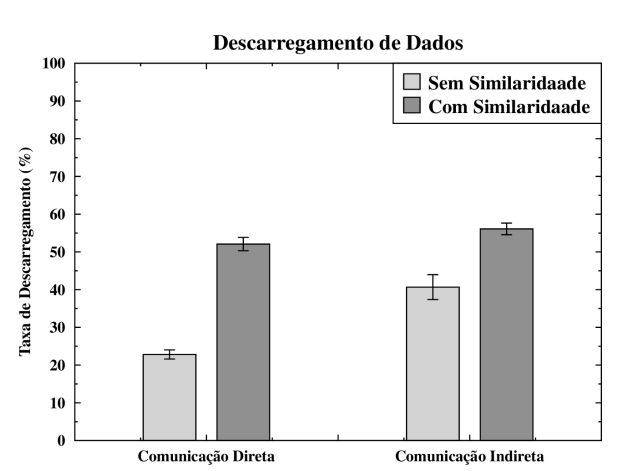

(a)

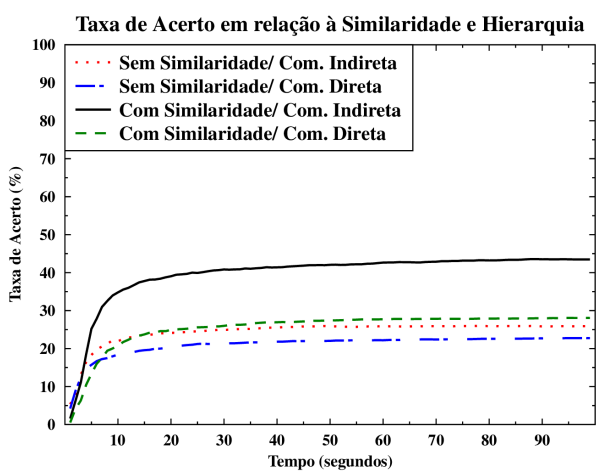

(b)

Figura 3. Influência da Similaridade e da Hierarquia no Descarregamento de Dados e na Taxa de Acerto.

Em relação a avaliação da taxa de acerto variando similaridade e comunicação, é possível observar na Figura 3(b), que os cenários de comunicação direta obtém taxa de acerto superior aos cenários de comunicação indireta. Isso acontece porque quando a comunicação é indireta, as SBCS recebem pacotes de interesse que não pertencem aos usuários do grupo que está associado a elas, portanto, a probabilidade de terem o conteúdo armazenado é menor. Apesar disso, o grupo de usuários associado a cada SCBS não sofre nenhum impacto decorrente dessa diminuição da taxa de acerto, visto que não há substituição em cache dos conteúdos que foram solicitados por outra SCBS. No entanto, pode haver um aumento no tempo de resposta ao usuário decorrente da comunicação indireta, visto que pode aumentar a quantidade de saltos para alcançar o conteúdo.

Alguns outros fatores que também podem influenciar na similaridade e na formação dos agrupamentos são a quantidade de dispositivos móveis na rede e a quantidade de conteúdos disponíveis. Ao analisar cenários em que há variação na quantidade de dispositivos móveis, foi observado que com 30 DMs, para cache de tamanho 20, é alcançada uma taxa de $40 \%$ de descarregamento de dados. Com um aumento de $50 \%$ na quantidade de nós, a degradação na taxa de descarregamento é de aproximadamente $5 \%$, resultando em valores estatisticamente semelhantes e indicando que a quantidade de dispositivos não tem alta interferência na taxa de descarregamento quando os interesses dos usuários continuam similares. Apesar disso, a adição de DMs pode causar aumento no uso da largura de banda, na perda de pacotes e no tempo de resposta ao usuário.

Em relação à variação na quantidade de conteúdos, foram avaliados cenários com 100 e 200 conteúdos. No cenário com 100 conteúdos, ao utilizar um tamanho de cache de $20 \%$ (cache $=20$ ), é possível obter uma taxa de descarregamento de, aproximadamente, $40 \%$. Com o mesmo tamanho de cache (cache $=20$ ), mas em um cenário com o dobro de conteúdos, há uma diminuição na taxa, que decai para a marca de $25 \%$. No entanto, para a nova quantidade de conteúdos, esse tamanho representa apenas $10 \%$ da quantidade total. Assim, mesmo com o dobro de conteúdos, foi possível obter uma taxa semelhante ao cenário sem similaridade com apenas 100 conteúdos. Ao avaliar o cenário com 200 conteúdos e tamanho de cache $=40$ é possível obter uma taxa de descarregamento de, aproximadamente, $45 \%$. Desta forma, observa-se que mesmo com o aumento na quanti- 
dade de conteúdos, ainda é possível manter a taxa de descarregamento, quando mantida a proporção no tamanho do cache.

\section{Conclusão}

De acordo com a avaliação de desempenho da estratégia, foi possível observar que considerar mais de um aspecto para medir a similaridade entre usuários pode gerar agrupamentos mais representativos, do ponto de vista de afinidade e, com isso, obter melhores resultados em relação a taxa de acerto e descarregamento de dados. Essas melhorias podem culminar em economia de recursos, melhoria de QoS e do tempo de resposta ao usuário. Além disso, o uso de um peso na função de similaridade da iSim permite que ela seja adaptável a diferentes cenários de acordo com as características da rede.

\section{Publicações}

A estrutura inicial do simulador foi apresentada no DCC DEMO DAY de 2016 sob o título "Gerenciamento de Cache Ciente de Similaridade em uma Rede MESH Centrada no Conteúdo" 1.

A iSim foi publicada na trilha principal do XXXV Simpósio Brasileiro de Redes de Computadores e Sistemas Distribuídos (SBRC) sob o título: Explorando a afinidade de usuários para descarregamento de dados mais eficiente em redes celulares de pequeno porte, com autoria de Adriana Viriato Ribeiro, Leobino N. Sampaio e Artur Ziviani. Além disso, foi aceita para publicação no IEEE Symposium on Computers and Communications (ISCC) de 2018 sob o título: Affinity-Based User Clustering for Efficient Edge Caching in Content-Centric Cellular Networks, também com autoria de Adriana Viriato Ribeiro, Leobino N. Sampaio e Artur Ziviani.

\section{Referências}

Chang, Z., Gu, Y., Han, Z., Chen, X., and Ristaniemi, T. (2016). Context-Aware Data Caching for 5G Heterogeneous Small Cells Networks. In Communications (ICC), 2016 IEEE International Conference on, pages 1-6. IEEE.

Chen, Z. and Kountouris, M. (2015). Cache-enabled Small Cell Networks with Local User Interest Correlation. In IEEE 16th International Workshop on Signal Processing Advances in Wireless Communications (SPAWC), pages 680-684.

ElBamby, M. S., Bennis, M., Saad, W., and Latva-Aho, M. (2014). Content-aware User Clustering and Caching in Wireless Small Cell Networks. In 11th International Symposium on Wireless Communications Systems (ISWCS), pages 945-949.

Hajri, S. E. and Assaad, M. (2016). Caching Improvement Using Adaptive User Clustering. arXiv preprint arXiv:1605.09602.

Ioannou, A. and Weber, S. (2016). A Survey of Caching Policies and Forwarding Mechanisms in Information-Centric Networking. IEEE Communications Surveys $\mathcal{E}$ Tutorials, pages $1553-877$.

Pantisano, F., Bennis, M., Saad, W., and Debbah, M. (2015). Match to Cache: Joint User Association and Backhaul Allocation in Cache-aware Small Cell Networks. In IEEE International Conference on Communications (ICC), pages 3082-3087.

\footnotetext{
${ }^{1}$ https://youtu.be/IPR5qpOLL8M
} 